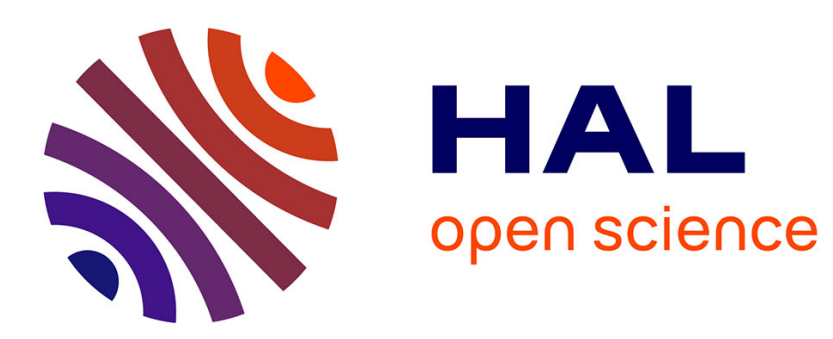

\title{
Distribution of local magnetisations in random networks of automata
}

\author{
Bernard Derrida, H Flyvbjerg
}

\section{To cite this version:}

Bernard Derrida, H Flyvbjerg. Distribution of local magnetisations in random networks of automata. Journal of Physics A: Mathematical and General, 1987, 20 (16), pp.L1107-L1112. 10.1088/03054470/20/16/013 . hal-03285907

\section{HAL Id: hal-03285907 \\ https://hal.science/hal-03285907}

Submitted on 19 Jul 2021

HAL is a multi-disciplinary open access archive for the deposit and dissemination of scientific research documents, whether they are published or not. The documents may come from teaching and research institutions in France or abroad, or from public or private research centers.
L'archive ouverte pluridisciplinaire HAL, est destinée au dépôt et à la diffusion de documents scientifiques de niveau recherche, publiés ou non, émanant des établissements d'enseignement et de recherche français ou étrangers, des laboratoires publics ou privés. 


\title{
LETTER TO THE EDITOR
}

\section{Distribution of local magnetisations in random networks of automata}

\author{
B Derrida $\uparrow+$ and H Flyvbjerg $\$ \|$ \\ $\dagger$ Institute for Theoretical Physics, University of California, Santa Barbara, CA 93106, USA \\ \$ Service de Physique Théorique, CEN Saclay, F-91191 Gif-sur-Yvette, France.
}

Received 7 September 1987

\begin{abstract}
We derive an integral equation which gives the distribution $P(m)$ of local magnetisations for a random network of automata (Kauffman model). We show how from this distribution one can recover the distances between configurations and the fraction of spins in the stable core (i.e. the spins which do not depend on the initial conditions). A numerical solution of the integral equation shows that $P(m)$ has singularities at $m=0$, $\pm \frac{1}{2}$ and \pm 1 .
\end{abstract}

The dynamics of neural networks and of automata have been much studied recently both by numerical and analytical methods (see Bienenstock et al (1986) for a recent review). Networks of automata can be defined as systems of $N$ Ising spins $S_{i}=-1$ or 1 which evolve according to

$$
S_{i}(t+1)=f_{i}\left(S_{j_{1}(i)}(t), \ldots, S_{j_{k}(i)}(t)\right) .
$$

A sample network is specified by giving each site $i$, a function $f_{i}$ of $k$ variables and its input sites $j_{1}(i), \ldots, j_{k}(i)$. Usually the number $k$ of inputs is the same for all sites $i$ but networks with a number of inputs depending on $i$ can also be considered.

Among all the possible models of automata (Wolfram 1983), there is a class of model (Kauffman 1969, 1970, 1974) for which some exact results concerning the time evolution can be obtained (Derrida and Weisbuch 1986, Hilhorst and Nijmeijer 1987, Flyvbjerg and Kjaer 1987). These automata are characterised by the fact that the inputs $j_{1}(i), \ldots, j_{k}(i)$ of each site $i$ are randomly chosen among the $N$ sites. Depending on the choice of the functions $f_{i}$, one can study the Kauffman model (Derrida and Weisbuch 1986), a non-symmetric spin glass (Derrida 1987a, b), neural network models (Derrida et al 1987, Kürten 1987). This class of model is soluble because the inputs $j_{1}(i), \ldots, j_{k}(i)$ are chosen at random. This allows one to show that some quantities (distances, magnetisations, projections on stored patterns) have exactly the same time evolution for the annealed model for which the functions $f_{i}$ and the inputs are changed at each time step and for the quenched model for which the functions $f_{i}$ and the inputs $j_{1}(i), \ldots, j_{k}(i)$ remain fixed (Derrida and Weisbuch 1986, Hilhorst and Nijmeijer 1987).

\footnotetext{
¥ Permanent address: Service de Physique Théorique, CEN Saclay, F-91191 Gif-sur-Yvette, France. Present address: The Niels Bohr Institute, University of Copenhagen, Blegdamsvej 17, DK-2100, Copenhagen $\varnothing$, Denmark.
} 
A system of $N$ automata has $2^{N}$ possible configurations. If one chooses an initial condition $C_{0}$

$$
C_{0}=\left\{S_{1}(0), \ldots, S_{N}(0)\right\}
$$

with a certain probability distribution $\rho_{0}\left(C_{0}\right)$, one can define an average magnetisation $m_{i}(t)$ of spin $i$ at time $t$

$$
m_{i}(t)=\left\langle S_{i}(t)\right\rangle
$$

where $S_{i}(t)$ is related to $S_{i}(0)$ by $(1)$ and where \langle\rangle means an average over initial conditions (configuration $C_{0}$ has a weight $\rho_{0}\left(C_{0}\right)$ ). The purpose of the present work is to show that, for the class of soluble models defined above, one can calculate the probability distribution $P_{t}(m)$ of local magnetisations defined by

$$
P_{t}(m)=\frac{1}{N} \sum_{i=1}^{N} \delta\left(m-m_{i}(t)\right) .
$$

Our results are valid under the following two conditions.

(i) The thermodynamic limit $N \rightarrow \infty$ is taken.

(ii) We restrict our calculations to the case of uncorrelated spins in the initial condition

$$
\rho_{0}\left(C_{0}\right)=\prod_{i=1}^{N} \frac{\left(1+m_{i}(0) S_{i}(0)\right)}{2} .
$$

If one wants to compute the value of a spin $S_{i}(t+1)$ at time $t+1$, one needs to know the whole tree of its $1+k+k^{2}+\ldots+k^{t+1}$ ancestors (its inputs at time $t$, the inputs of its inputs at time $t-1$, and so on up to time $t=0$ ). Because all these inputs are chosen at random among the $N$ sites, for almost all sites $i$, all the sites in the tree of its ancestors are different if $N \rightarrow \infty$ (Derrida and Weisbuch 1986). Therefore, for almost all sites $i$, its inputs $j_{1}(i), \ldots, j_{k}(i)$ at time $t$ are uncorrelated and the probability that these inputs take the values $S_{j_{1}}, \ldots, S_{j k}$ can be factorised:

$$
\left(\frac{1+m_{j_{1}}(t) S_{j_{1}}}{2}\right) \ldots\left(\frac{1+m_{j_{k}}(t) S_{j_{k}}}{2}\right)
$$

Therefore for almost all sites $i$ (i.e. in the limit $N \rightarrow \infty$ ), $m_{i}(t)$ is given by

$$
m_{i}(t)=\sum_{s_{i}= \pm 1} \ldots \sum_{s_{i_{k}}= \pm 1}\left(\frac{1+m_{j_{1}}(t) S_{j_{1}}}{2}\right) \ldots\left(\frac{1+m_{j_{k}}(t) S_{j_{k}}}{2}\right) f_{i}\left(S_{j_{1}}, \ldots, S_{j_{k}}\right) \text {. }
$$

Since $(7)$ is true for almost all sites $i$, one can write the following recursion relations for $P_{1}(m)$

$$
\begin{aligned}
P_{t+1}(m)=\sum_{f} Q & (f) \int \ldots \int \mathrm{d} m_{1} \ldots \mathrm{d} m_{k} P_{l}\left(m_{1}\right) \ldots P_{l}\left(m_{k}\right) \\
& =\delta\left[m-\sum_{S_{1}= \pm 1} \ldots \sum_{S_{h}= \pm 1}\left(\frac{1+m_{1} S_{1}}{2}\right) \ldots\left(\frac{1+m_{k} S_{k}}{2}\right) f\left(S_{1}, \ldots S_{k}\right)\right]
\end{aligned}
$$

where the sum over $f$ is the sum over all the possible Boolean functions $f$ of $k$ variables and $Q(f)$ is the probability of the Boolean function $f$. The average over $Q(f)$ comes from the fact that, in the limit $N \rightarrow \infty$, there are $N Q(f)$ sites $i$ which have a certain function $f$. 
One should notice that, depending on $Q(f), P_{t}(m)$ may or may not have a limiting value in the long-time limit and that this limit may depend on the initial condition $P_{0}(m)$. It is also important to notice that the condition (5) that the spins $S_{i}$ are uncorrelated in the initial configuration was essential to derive (7) and (8).

In the following we will restrict our discussion to the Kauffman model (1969) which is characterised by the fact that all the Boolean functions of $k$ variables have the same probability $\left(Q(f)=2^{-2^{h}}\right)$. One can, however, easily generalise the results to other cases (neural networks, non-symmetric spin glasses, etc).

The knowledge of $P_{1}(m)$ allows the calculation of several interesting properties of the model. For example, one can calculate the generalised distance $x_{n}$ between $n$ configurations. If one chooses at time $t=0, n$ random initial configurations with the probability (5), one can define $N x_{n}(t)$ as the number of spins $i$ which are identical in the $n$ configurations at time $t$. If $P_{t}(m)$ is known, then $x_{n}(t)$ is given by

$$
x_{n}(t)=\int \mathrm{d} m P_{t}(m)\left[\left(\frac{1+m}{2}\right)^{n}+\left(\frac{1-m}{2}\right)^{n}\right] \text {. }
$$

This formula can easily be understood. If site $i$ has magnetisation $m_{i}(t)$, this means that for a randomly chosen initial configuration $S_{i}(t)=+1$ with probability $\left(1+m_{i}(t)\right) / 2$ and -1 with probability $\left(1-m_{i}(t)\right) / 2$. The probability that site $i$ is identical in the $n$ configurations is therefore $\left[\left(1+m_{i}(t)\right) / 2\right]^{n}+\left[\left(1-m_{i}(t)\right) / 2\right]^{n}$.

So we see that the information contained in $x_{1}, x_{2}, \ldots, x_{n}$ is the same as in the first $n$ moments of $P_{t}(m)$. For the Kauffman model, it had been shown (Derrida and Weisbuch 1986) that the $x_{i}$ for odd $i$ are related to the even ones and that the time evolution of $x_{2}$ and $x_{4}$ is given by

$$
\begin{aligned}
& x_{2}(t+1)=\frac{1}{2}\left[1+\left(x_{2}(t)\right)^{k}\right] \\
& x_{4}(t+1)=\frac{1}{8}+\frac{3}{4}\left(x_{2}(t)\right)^{k}+\frac{3}{8}\left(1-2 x_{2}(t)+2 x_{4}(t)\right)^{k}-\frac{1}{4}\left(x_{4}(t)\right)^{k} .
\end{aligned}
$$

From (7) and (8) one can compute the moments $\mu_{n}(t)$ of $P_{t}(m)$

$$
\mu_{n}(t)=\int \mathrm{d} m P_{\mathrm{t}}(m) m^{n}
$$

and one finds

$$
\begin{aligned}
& \mu_{2}(t+1)=\left(\frac{1+\mu_{2}(t)}{2}\right)^{k} \\
& \mu_{4}(t+1)= 3\left(\frac{1+2 \mu_{2}(t)+\mu_{4}(t)}{4}\right)^{k}-2\left(\frac{1+6 \mu_{2}(t)+\mu_{4}(t)}{8}\right)^{k} \\
& \mu_{6}(t+1)= 16\left(\frac{1+15 \mu_{2}(t)+15 \mu_{4}(t)+\mu_{6}(t)}{32}\right)^{k}-30\left(\frac{1+7 \mu_{2}(t)+7 \mu_{4}(t)+\mu_{6}(t)}{16}\right)^{k} \\
&+15\left(\frac{1+3 \mu_{2}(t)+3 \mu_{4}(t)+\mu_{6}(t)}{8}\right)^{k}
\end{aligned}
$$

Of course from (9) one can easily check that $(12 a)$ and $(12 b)$ are equivalent to $(10 a)$ and $(10 b)$. We see that the integral equation (8) allows the calculation of all the moments $\mu_{n}$ and $x_{n}$. In particular, it allows the calculation of the stable core $s(t)$ which is the fraction of spins at time $t$ which do not depend on the initial condition

$$
s(t)=\lim _{n \rightarrow \infty} x_{n}(t)=\lim _{p \rightarrow \infty} \mu_{2 p}(t) .
$$


$s(t)$ is nothing but the fraction of spins which are such that $m_{i}(t)=+1$ or $m_{i}(t)=-1$. Using the recursion (8) and separating the contribution of the delta functions at +1 and -1 in $P_{1}(m)$

$$
P_{t}(m)=\frac{1}{2} s(t)[\delta(m-1)+\delta(m+1)]+\tilde{P}_{t}(m)
$$

one obtains for the time evolution of $s(t)$ :

$$
s(t+1)=\sum_{p=0}^{k} \frac{k !}{(k-p) ! p !}(s(t))^{k-p}(1-s(t))^{p} 2^{1-2^{r}} .
$$

This formula can easily be derived directly (Flyvbjerg 1987) by noticing that the $p$ th term in the sum (15) corresponds to a site $i$ which has $k-p$ inputs in the stable core at time $t$. For this spin $i$ to be in the stable core at time $t+1$, one needs that the function $f_{i}$ does not depend on the $p$ remaining spins. The probability of such a function $f_{i}$ is $2^{1-2^{r}}$.

In principle the shape of $P_{t}(m)$ can be obtained from the integral equation (8). In practice, the calculation of the $\mu_{n}$ becomes more and more difficult as $n$ increases. In order to obtain the shape of $P_{1}(m)$ in the limit $t \rightarrow \infty$, we used a Monte Carlo method. This method is nothing but the simulation of the annealed version of the Kauffman model for which $P_{t}(m)$ is also given by (8). We considered a sample of $N$ values of $m_{i}$ chosen at random at $t=0$. At each time step, we choose for each $i$ a new function $f_{i}$ and $k$ inputs at random and then we use (7) to compute the new $m_{i}$. We did our calculations for $k=3$ both for $N=10^{3}$ spins with $10^{4}$ iterations and for $N=10^{4}$ spins with $10^{3}$ iterations. The results were identical within statistical errors. In both cases $P_{\infty}(m)$ was obtained as the histogram of the $m_{i}$ of the $10^{7}$ sites. All these calculations were done after a 'thermalisation time' of typically $10^{3}-10^{4}$ iterations (to get the limit $t \rightarrow \infty$ and thus) to eliminate the dependence on initial conditions. From these simulations we obtained estimates for the moments $\mu_{2}(\infty), \mu_{4}(\infty), \mu_{6}(\infty)$ and for the stable core $s(\infty)$ in excellent agreement with the fixed points of (12) and (15). For example we obtained $s(\infty)=0.012196 \pm 0.00003$ by this Monte Carlo procedure whereas the fixed point of (15) for $k=3$ is 0.0122261 . We obtained also the shape of $\tilde{P}_{\propto}(m)$, i.e. the part of $P_{x}(m)$ which does not include the $\delta$ functions at \pm 1 , as shown in figure 1 .

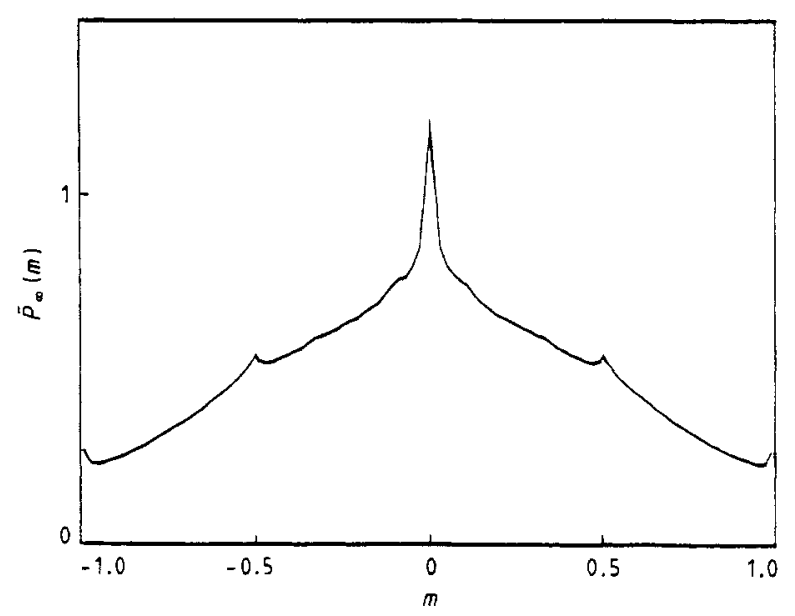

Figure 1. The continuous part of $P_{\infty}(m)$ for the Kauffman model for $k=3$. 
We see that, in addition to the $\delta$ functions at $m=-1$ and $1, P(m)$ exhibits visible singularities at $m=0, m= \pm \frac{1}{2}$ and $m= \pm 1$. We think that these singularities at $m=0$, $\pm \frac{1}{2}$ and \pm 1 are not delta functions because they did not change when we increased the number of time steps, the system size or the number of bins to describe our histogram. Thus $P_{\infty}(m)$ has a rather complex structure which becomes even more complicated in finite dimension (Derrida 1986).

We did not find a way of calculating analytically the shapes and the locations of these singularities from the integral equation (8). However such singularities in $P(m)$ are not very surprising since similar singularities have already been found and explained in solutions of simple integral equations (Derrida and Flyvbjerg 1987).

In this letter we have seen how one can write an integral equation $(8)$ for $P(m)$. We have also seen how from this integral equation one can deduce the fraction of spins in the stable core, i.e. the spins which do not depend on the initial conditions. These calculations could easily be generalised to other problems like non-symmetric spin glasses, neural network models, etc. The only thing to change would be the weights $Q(f)$ of the Boolean functions in the integral equation (8).

To compute $P(m)$, it was necessary to start with a distribution (5) of initial conditions for which the spins are uncorrelated. It would of course be interesting to investigate the case of correlated spins in the initial conditions. It would also be interesting to understand the origin of the visible singularities at $m=0, \pm \frac{1}{2}$ and \pm 1 in $P(m)$. Integral equations similar to $(8)$ have already been considered in the study of spin glasses on Bethe lattices (Bowman and Levin 1982, Thouless 1986). In that problem too, it has been recently suggested that correlations in the boundary conditions (which play the role of initial conditions in automata problems) could have an important effect (Mottishaw 1987).

The shape of $P(m)$ (delta functions and continuous part) is also very reminiscent of the distribution of local fields in the mean field theory of spin glasses with finite connectivity (Orland 1985, Viana and Bray 1985, Kanter and Sompolinsky 1987, De Dominicis and Mottishaw 1987, Mezard and Parisi 1987). This strengthens the idea that spin glasses and automata have many features in common (Derrida and Flyvbjerg 1986).

We thank $P$ Mottishaw for discussions about the relationship with spin glasses on Bethe lattices. HF thanks the Service de Physique Théorique at Saclay for its hospitality. This research was supported in part by the National Science Foundation under Grant no PHY82-17853, supplemented by funds from the National Aeronautics and Space Administration, at the University of California at Santa Barbara.

\section{References}

Bienenstock E, Fogelman Souliè F and Weisbuch G (ed) 1986 Disordered Systems and Biological Organization (Berlin: Springer)

Bowman D R and Levin K 1982 Phys. Rev. B 253438

De Dominicis C and Mottishaw P 1987 Europhys. Lett. 387

Derrida B 1987a J. Phys. A: Math. Gen. 20 L721

— 1987b Chance and Matter, Les Houches $1986 \mathrm{ed} J$ Souletie, J Vannimenus and R Stora to be published Derrida B and Flyvbjerg H 1986 J. Phys. A: Maih. Gen. 19 L1003

1987 J. Phys. A: Math. Gen. 205273

Derrida B, Gardner E and Zippelius A 1987 Europhys. Lett. 4167 
Derrida B and Weisbuch G 1986 J. Physique 471297

Flyvbjerg H 1987 Phys. Scr. to be published

Flyvbjerg $H$ and Kjaer N J 1987 J. Phys. A: Math. Gen. submitted

Hilhorst $H$ and Nijmeijer M $1987 J$. Physique 4885

Kanter I and Sompolinsky H 1987 Phys. Rev. Lett. 58164

Kauffman S A 1969 J. Theor. Biol. 22437

1970 Math. Life Sci. 363

1974 J. Theor. Biol. 44167

Kürten K 1987 Preprint

Mezard M and Parisi G 1987 Europhys. Lett. 31067

Mottishaw P 1987 Europhys. Lett. 4333

Orland H 1985 J. Physique Lett. 46 L763

Thouless D J 1986 Phys. Rev. Lett. 561082

Viana L and Bray A J 1985 J. Phys. C: Solid State Phys. 183037

Wolfram S 1983 Rev. Mod. Phys. 55601 